Check for updates

Cite this: RSC Adv., 2020, 10, 26862

Received 12th May 2020

Accepted 9th July 2020

DOI: 10.1039/d0ra04250a

rsc.li/rsc-advances

\section{Proteomics and metabolomics analysis reveal potential mechanism of extended-spectrum $\beta$ - lactamase production in Escherichia coli $\uparrow$}

\begin{abstract}
He Ma, (DD ${ }^{\text {ab }}$ Bingjie Lai, ${ }^{c}$ Yufen Jin, ${ }^{d}$ Chang Tian, ${ }^{a}$ Jiaying Liu ${ }^{a}$ and Ke Wang ${ }^{\star a}$
In this study, ten clinical susceptible strains and ten clinical ESBL-EC (extended-spectrum $\beta$-lactamaseproducing Escherichia coli) were screened and obtained by microbial identification using ITEK® 2 Compact. TMT (Tandem Mass Tag) proteomics analysis discovered 1553 DEPs (differentially expressed proteins) between ESBL-EC and non-ESBL-EC. In addition, an untargeted metabolomics assay by using UHPLC-MS (ultra-high-performance liquid chromatography-mass spectrometry) was applied to compare the differential profiles of metabolites between $\beta$-lactam antibiotic-sensitive $E$. coli and multidrug-resistant ESBL-producing E. coli strains. The PCA (principal component analysis) score plots and OPLS-DA (orthogonal projections to latent structures discriminant analysis) plots clearly discriminated ESBL-EC and non-ESBL-EC, and volcano analysis presented 606 and 459 altered metabolites between ESBL-EC vs. non-ESBL-EC in positive and negative ion modes, respectively. Interestingly, the bioinformatics analysis demonstrated that the purine metabolism pathway was enriched in ESBL-EC. These results suggest that the existence of extended-spectrum $\beta$-lactamase affects the metabolite and protein profiles of $E$. coli. The correlation analysis of metabolomics and proteomics data established a correlation between DEPs and differential metabolites in the purine metabolism pathway. Moreover, three metabolite candidates in the purine metabolism pathway were validated by the UPLCMRM-MS (ultra-performance liquid chromatography multiple reaction monitoring mass spectrometry) method. Our data suggest that these DEPs and differential metabolites may play important roles in the antibiotic resistance of ESBL-EC. Our study can provide scientific data for the mechanism study of antibiotic resistance of ESBL-EC at the metabolite and protein levels and targeting modulators to these pathways may be effective for treatment of ESBL-EC strains.
\end{abstract}

\section{Introduction}

E. coli (Escherichia coli) has the characteristics of being both a widespread gut commensal of vertebrates and a versatile pathogen. ${ }^{1}$ E. coli is a common cause of urinary tract infection and intra-abdominal infection in humans of all ages, ${ }^{2}$ and it kills more than 2 million humans annually in the form of intraand extra-intestinal diseases. ${ }^{3,4}$ ESBLs (Extended-spectrum $\beta$ lactamases) are plasmid-encoded $\beta$-lactamases resistant to penicillins, cephalosporins, $\beta$-lactams, and other antimicrobials, such as aminoglycosides and fluoroquinolones. ${ }^{5}$ The

${ }^{a}$ Department of Respiratory Medicine, The Second Hospital of Jilin University, Changchun, China.E-mail: kewangm1@hotmail.com

${ }^{b}$ Department of Anesthesiology, The Second Hospital of Jilin University, Changchun, China

${ }^{c}$ Department of Intensive Care Unit, The Second Hospital of Jilin University, Changchun, China

${ }^{d}$ Clinical Laboratory, The Second Hospital of Jilin University, Changchun, China

$\dagger$ Electronic supplementary information (ESI) available. See DOI: 10.1039/d0ra04250a emergence of multidrug-resistant bacteria renders the treatment of bacterial infections an urgent global challenge. ${ }^{6}$ Thus, the mechanisms of $E$. coli resistance should be clearly understood in the future development of antibiotics, and new therapy methods should be sought.

Detecting and identifying metabolome-scale changes in microorganisms is essential in understanding their roles in cellular processes. Metabolites may be used to realize the molecular basis for a biological phenomenon, including cell signaling. ${ }^{7}$ Metabolomics, one of the most powerful and promising tools for analyzing metabolism, ${ }^{8}$ is a highthroughput analytical technology used to identify and quantify small amounts of metabolites. ${ }^{9}$ This tool provides a broad range of information on integrated cellular response. ${ }^{\mathbf{1 0}}$ Furthermore, metabolomics has been applied to a variety of pathophysiological process, including cancer and diabetes, with the goal of identifying the biomarkers predictive of a disease onset and ensuring treatment efficacy monitoring. ${ }^{11}$ Studies have been reported on metabolomics techniques that focus on the role of bacterial metabolism in constraining the evolution of antibiotic resistance. ${ }^{12}$ Wei et al. characterized the 
mechanisms of $p$-aminosalicylic acid resistance in Mycobacterium tuberculosis might be associated with the increased metabolites level in phenylalanine metabolism pathway using multi-omics (genome, proteome, and metabolome) analysis. ${ }^{13}$ Zhao et al. used multi-omics (LC-MS based metabolomic, and iTRAQ labeling proteomic) analysis to assess capreomycin resistance on tlyA deficient and point mutation (G695A) Mycobacterium tuberculosis strains, with the finding that the greater drug tolerance of CAPr1 strains may be associated with the weakening of $S$-adenosyl-L-methionine-dependent methyltransferase activity and abnormal membrane lipid metabolism. $^{\mathbf{1 4}}$

In addition to metabolomics, proteomics is also a potent tool for improving the characterization of complex biological systems or pathologies. Proteomics methods have been used to identify protein network alterations, thus helping researchers understand the responses to various diseases. ${ }^{15,16}$ Protein expression studies based on proteomics have unique advantages, such as large-scale, high-throughput, high-sensitivity, and dynamic formats. ${ }^{17}$ The proteomics methods for several types of drug resistance have attracted increasing attention in previous studies. ${ }^{18}$ Investigating metabolome-scale changes may provide insights into the widespread modes of acquired bacterial resistance in clinics. ${ }^{7}$

In this study, a conjoint analysis of TMT (Tandem Mass Tag) proteomics and metabolomics was applied to compare the differential profile of proteins and metabolites between $\beta$-lactam antibiotic-sensitive $E$. coli and multidrug-resistant ESBLproducing $E$. coli strains and reveal the mechanisms of antibiotic resistance of clinical $E$. coli strains. The novelty of this study lies in revealing the metabolic characteristics of enzymeproducing bacteria and finding potential important pathways and genes for regulating enzyme production.

\section{Materials and methods}

\subsection{Chemicals and reagents}

Ultrapure water was produced by a Ming Che D24 UV water purification system (Merck Millipore, Burlington, USA). $\mathrm{MeOH}$, ACN, $\mathrm{NH}_{4} \mathrm{OAc}$, and $\mathrm{NH}_{4} \mathrm{OH}$ (CNW Technologies, Düsseldorf, Germany) were all MS grade. 2-Chloro-L-phenylalanine (Sigma, St. Louis, MO, USA) was used as an internal standard. HEPES, SDS, TFA, triethylammonium bicarbonate (TEAB), ammonium formate, Columbia agar, MacConkey agar, and Chocolate agar were obtained from Sigma (St. Louis, MO, USA). Dithiothreitol (DTT), iodoacetamide (IAA), and trypsin (sequencing grade) were obtained from Promega Life Sciences (Madison, USA). Urea was provided by GibcoBRL (MD, USA). EDTA and PMSF were obtained from Amesco (USA). Ethanol, formic acid, acetone, BCA protein quantification kit, TMT 6 standard kit, Acclaim PepMap C18 Column $(100 \mu \mathrm{m} \times 20 \mathrm{~mm}, 75 \mu \mathrm{m} \times 250$ $\mathrm{mm}$ ) were purchased from Thermo Fisher Scientific (Waltham, MA, USA). TEAB and 25\% ammonia were obtained from Santa Cruz (CA, USA). Sep Pak $\mathrm{C}_{18}$ cartridges $(1 \mathrm{cc}, 100 \mathrm{mg}$ ) and the Acquity UPLC®BEH C18 column $(50 \mathrm{~mm} \times 2.1 \mathrm{~mm}, 1.7 \mu \mathrm{m})$ were obtained from Waters (Milford, USA). The Luria-Bertani (LB) broth and LB agar were purchased from Becton Dickinson
(Sparks, MD, USA). The lysis buffer for protein analysis was obtained from Roche Ltd (Basel, Switzerland). All other reagents were of ACS reagent grade.

\subsection{Clinical bacterial specimens collection and cultivation}

Clinical specimens of human phlegm, urine, blood, secretions were randomly collected on January-June 2018 from patients of the Second Hospital of Jilin University, the specimens were divided as experimental group $(n=10)$ and control group $(n=$ 10). Detailed sample information was list in Table 1. The patients signed informed consent forms for the experimental study. All experimental procedures were performed in accordance with the Guidelines for the Collection and Application of Human Related Specimens of the Second Hospital of Jilin University and approved by the Ethics Committee of the Second Hospital of Jilin University. The age and gender of all patients included in this research were recorded. The phlegm samples must comply with the requirements of more than 25 leukocytes and less than 10 epithelial cells in the low-power field. The phlegm and urine samples were planted with Columbia agar with blood solution, MacConkey agar, and Chocolate agar plates and kept in a $5 \% \mathrm{CO}_{2}$ incubator at $35{ }^{\circ} \mathrm{C}$. Blood samples were cultured in automated blood culture flask system. The blood samples with bacterial infection were used for further plate culture. A single colony was selected and cultured in the LB broth to 0.5 MCF. After microbial identification and sensitivity analysis with the aid of VITEK 2 Compact, the bacterial suspension was coated on a plate, then ceftazidime, ceftazidime/clavulanic acid and cefotaxime, and cefotaxime/ clavulanic acid were used as indicators to detect the ESBLs in the E. coli according to the CLSI (2014) Performance Standards for Antimicrobial Susceptibility Testing.

\subsection{Proteomics analysis}

2.3.1. Proteomics sample preparation. Each sample was mixed with $1 \mathrm{~mL}$ of lysis buffer and subjected to sonication on ice for $10 \mathrm{~min}$. After centrifugation at $20000 \times \mathrm{g}$ for $30 \mathrm{~min}$ at $4{ }^{\circ} \mathrm{C}$, the supernatant was collected and measured for protein concentration with BCA. Then, the $100 \mu \mathrm{g}$ protein was selected and set to the constant volume of $100 \mu \mathrm{L}$ per test with $100 \mathrm{mM}$ of TEAB.

2.3.2. Protein digestion and TMT labeling. Each sample was co-incubated with $5 \mu \mathrm{L}$ of DTT $(200 \mathrm{mM})$ for $1 \mathrm{~h}$ at $55^{\circ} \mathrm{C}$, followed by $30 \mathrm{~min}$ for the RT co-incubation of $5 \mu \mathrm{L}$ of IAA $(375$ $\mathrm{mM})$. After the precipitation with cold acetone, the sample was dissolved with $100 \mu \mathrm{L}$ of TEAB and subjected to trypsin digestion. The digested peptide product was labeled using TMT kits. The sample was ready for separation after desalting and vacuum drying.

2.3.3. Low $\mathbf{p H}$ nano-HPLC-MS/MS analysis and data processing. After high $\mathrm{pH}$ reverse phase separation of the sample, the fractions were resuspended with $40 \mu \mathrm{L}$ solvent $\mathrm{C}$ respectively (C: water with $0.1 \%$ formic acid; D: ACN with $0.1 \%$ formic acid), separated by nanoLC and analyzed by on-line electrospray tandem mass spectrometry. The experiments were performed on a Nano Aquity UPLC system (Waters Corporation, Milford, 
Table 1 The paralleled collection times for clinical bacterial specimens

\begin{tabular}{|c|c|c|c|c|c|c|}
\hline Sample number & Experimental group & Collection time & Gender & $\begin{array}{l}\text { Control } \\
\text { group }\end{array}$ & Collection time & Gender \\
\hline 1 & Urine & $2018 / 1 / 25$ & Male & Urine & $2018 / 2 / 15$ & Male \\
\hline 2 & Blood & $2018 / 3 / 1$ & Male & Phlegm & $2018 / 3 / 9$ & Female \\
\hline 3 & Blood & $2018 / 3 / 12$ & Female & Phlegm & $2018 / 3 / 21$ & Female \\
\hline 4 & Phlegm & $2018 / 3 / 29$ & Male & Blood & $2018 / 3 / 27$ & Male \\
\hline 5 & Phlegm & $2018 / 4 / 20$ & Female & Blood & $2018 / 4 / 1$ & Female \\
\hline 6 & Blood & $2018 / 5 / 3$ & Female & Urine & $2018 / 4 / 27$ & Male \\
\hline 7 & Urine & $2018 / 5 / 12$ & Male & Blood & $2018 / 5 / 6$ & Male \\
\hline 8 & Phlegm & $2018 / 5 / 20$ & Female & Urine & $2018 / 5 / 24$ & Female \\
\hline 9 & Urine & $2018 / 5 / 21$ & Female & Phlegm & $2018 / 6 / 1$ & Male \\
\hline 10 & Vaginal secretion & $2018 / 5 / 27$ & Female & Phlegm & $2018 / 6 / 5$ & Female \\
\hline
\end{tabular}

MA) connected to a quadrupole-Orbitrap mass spectrometer (QExactive) (Thermo Fisher Scientific, Bremen, Germany) equipped with an online nano-electrospray ion source. $4 \mu \mathrm{L}$ peptide sample was loaded onto the trap column (Thermo Scientific Acclaim PepMap C18, $100 \mu \mathrm{m} \times 2 \mathrm{~cm}$ ), with a flow of 10 $\mu \mathrm{L} \min ^{-1}$ for $3 \mathrm{~min}$ and subsequently separated on the analytical column (Acclaim PepMap C18, $75 \mu \mathrm{m} \times 25 \mathrm{~cm}$ ) with a linear gradient, from $5 \% \mathrm{D}$ to $30 \% \mathrm{D}$ in $80 \mathrm{~min}$. The column was cleaned then was re-equilibrated at initial conditions for $10 \mathrm{~min}$. The column flow rate was maintained at $300 \mathrm{~nL} \mathrm{~min}{ }^{-1}$ and column temperature was maintained at $45{ }^{\circ} \mathrm{C}$. The electrospray voltage of $2.0 \mathrm{kV}$ versus the inlet of the mass spectrometer was used. The Q-Exactive mass spectrometer was operated in the data-dependent mode to switch automatically between MS and MS/MS acquisition. Survey full-scan MS spectra $(\mathrm{m} / \mathrm{z} 350-1600)$ were acquired with a mass resolution of $70 \mathrm{~K}$, followed by fifteen sequential high energy collisional dissociation (HCD) MS/MS scans with a resolution of $17.5 \mathrm{~K}$. In all cases, one microscan was recorded using dynamic exclusion of 30 seconds. MS/MS fixed first mass was set at 100. Tandem mass spectra were then subjected to the proteome database searching and quantitative data analysis as described in the ESI. $\dagger^{19}$

\subsection{Metabolomics analysis}

2.4.1. Metabolites extraction. After the centrifugation of the bacterial culture solution at $3000 \times g$ for $15 \mathrm{~min}$ at $4{ }^{\circ} \mathrm{C}$, the $50 \mathrm{mg}$ pellets were taken and suspended in $1000 \mu \mathrm{L}$ of the cold extraction solution ( $40 \% \mathrm{MeOH}, 40 \%$ acetic acid, and $20 \% \mathrm{H}_{2} \mathrm{O}$ ) containing an internal standard (2-chloro-L-phenylalanine). The suspension was homogenized with a ball mill at $40 \mathrm{~Hz}$ for $5 \mathrm{~min}$ and subjected to $5 \mathrm{~min}$ of ultrasonic water bath, which was repeated three times, then the protein was precipitated by incubation for $1 \mathrm{~h}$ at $-20{ }^{\circ} \mathrm{C}$. After centrifugation at $3000 \times \mathrm{g}$ for $15 \mathrm{~min}$ at $4{ }^{\circ} \mathrm{C}$, the $825 \mu \mathrm{L}$ supernatant was collected and vacuum-dried. The dried metabolite sample was reconstituted with $100 \mu \mathrm{L}$ of the extraction solution $\left(50 \% \mathrm{ACN}\right.$ and $\left.50 \% \mathrm{H}_{2} \mathrm{O}\right)$, vortexed for $30 \mathrm{~s}$, subjected to $10 \mathrm{~min}$ of ultrasonic water bath at $4{ }^{\circ} \mathrm{C}$, and centrifuged at $3000 \times g$ for $15 \mathrm{~min}$ at $4{ }^{\circ} \mathrm{C}$. The $60 \mu \mathrm{L}$ supernatant was collected and prepared for UHPLC-QTOF-MS analysis. The $10 \mu \mathrm{L}$ supernatant was collected from each sample and combined as the QC sample.
A

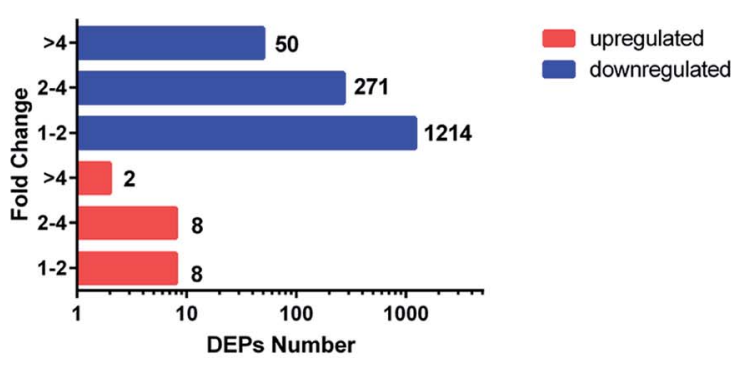

B

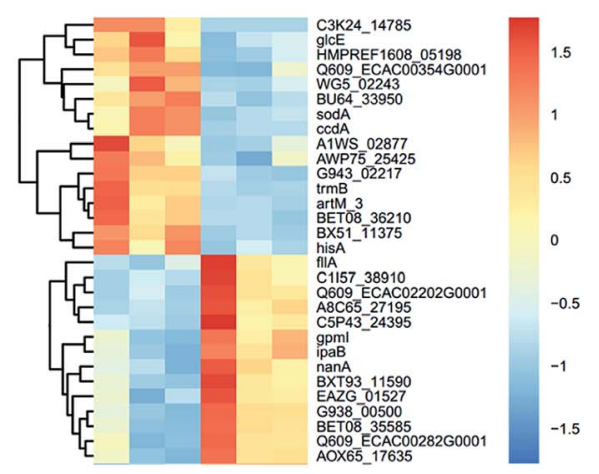

Fig. 1 (A) Number of proteins identified as upregulated and downregulated between ESBL-EC and non-ESBL-EC. The red bars represent the upregulated proteins. The blue bars represent the downregulated proteins. The $x$-axis represents the number of DEPs. The $y$-axis represents the fold change value. (B) Heatmap based on representative hierarchical clustering analysis with 30 DEPs between ESBL-EC and non-ESBL-EC (the detailed heatmap is shown in Fig. S1 $\uparrow$ ). The relative protein level is depicted in color scale, in which red indicates upregulation, and blue indicates downregulation. 


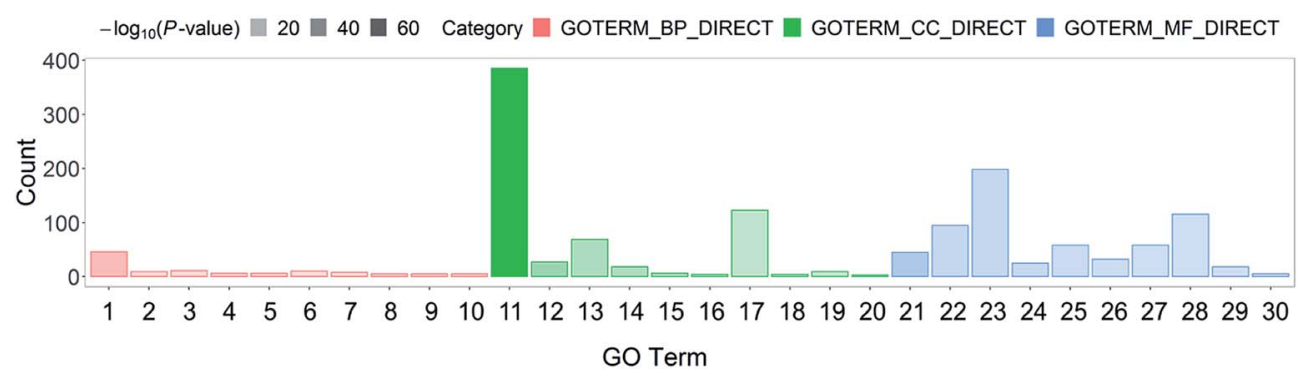

Fig. 2 Top $10 \mathrm{GO}$ classification histograms of DEPs for ESBL-EC vs. non-ESBL-EC. The $x$-axis represents GO_BP (GO Term Biological Process), GO_CC (Cellular Component), and GO_MF (Molecular Function). (1) Translation, (2) transcription antitermination, (3) glycolytic process, (4) isopentenyl diphosphate biosynthetic process, methylerythritol 4-phosphate pathway, (5) negative regulation of translational initiation, (6) nucleobase-containing small molecule interconversion, (7) ubiquinone biosynthetic process, (8) nucleotide phosphorylation, (9) terpenoid biosynthetic process, (10) DNA-templated transcription, termination, (11) cytosol, (12) cytosolic large ribosomal subunit, (13) membrane, (14) cytosolic small ribosomal subunit, (15) chromosome, (16) DNA topoisomerase complex (ATP-hydrolyzing), (17) cytoplasm, (18) glycerol-3phosphate dehydrogenase complex, (19) cell division site, (20) cell surface, (21) structural constituent of ribosome, (22) identical protein binding, (23) protein binding, (24) rRNA binding, (25) zinc ion binding, (26) RNA binding, (27) magnesium ion binding, (28) ATP binding, (29) tRNA binding, (30) mRNA 5'-UTR binding. The $y$-axis represents the number of involved DEPs between ESBL-EC and non-ESBL-EC.

2.4.2. UHPLC-MS (ultra-high-performance liquid chromatography-mass spectrometry) analysis. For the untargeted metabolomics analysis, UHPLC-MS analyses were performed using an UHPLC system (1290, Agilent Technologies) with a UPLC BEH Amide column $(1.7 \mu \mathrm{m}, 2.1 \times 100 \mathrm{~mm}$, Waters) coupled to Triple TOF 5600 (Q-TOF, AB Sciex). The
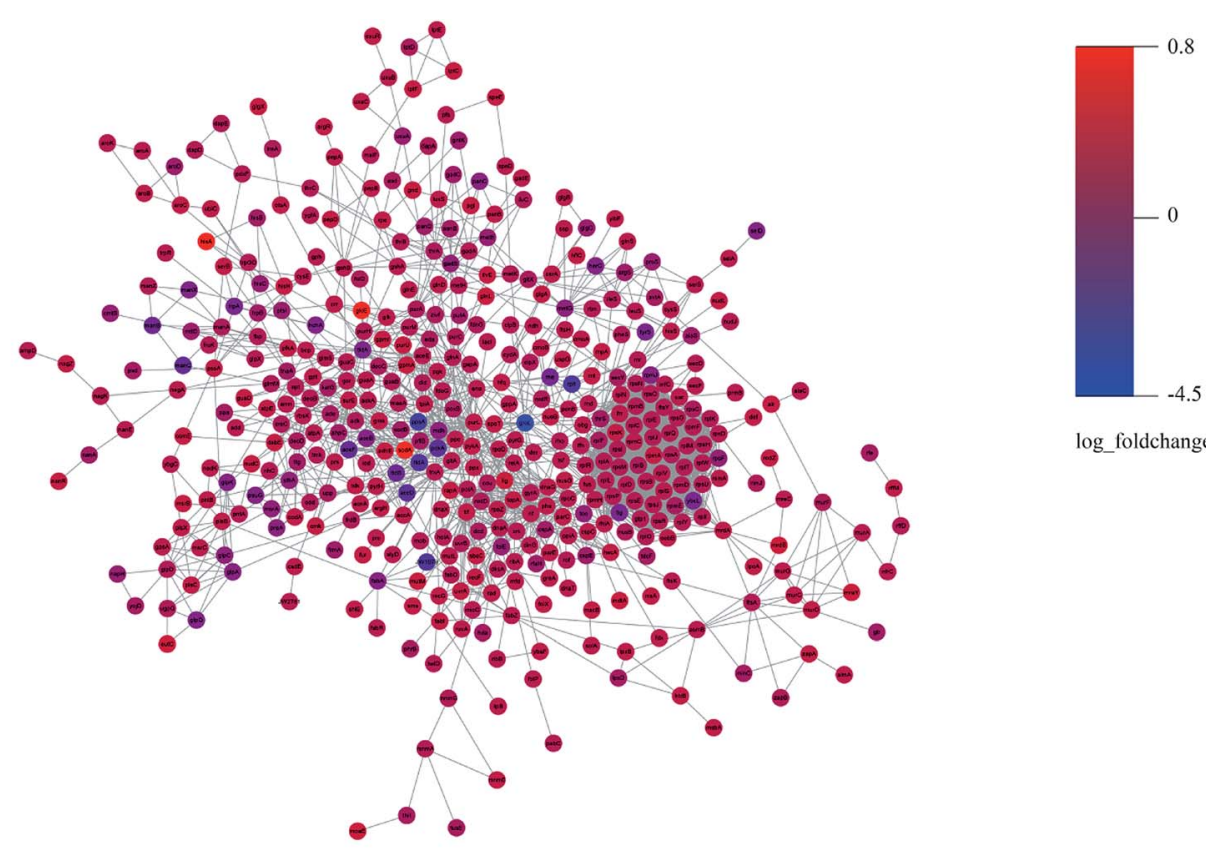

$\log$ foldchange
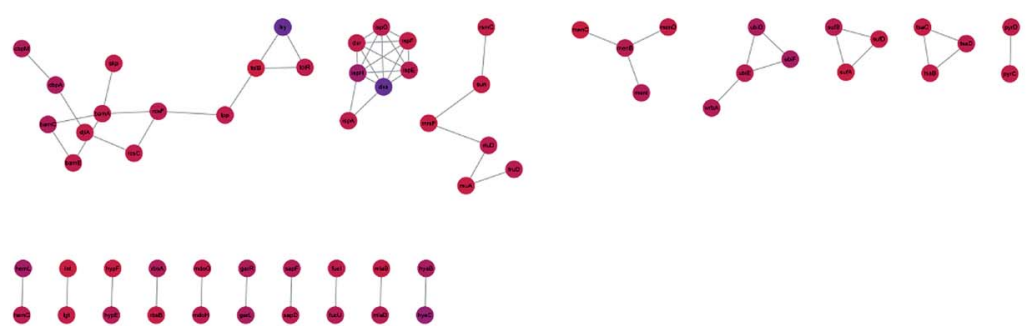

Fig. 3 PPI (protein-protein interaction) network of DEPs between ESBL-EC and non-ESBL-EC. Large sizes and dark colors represent the high impact and level of DEPs. 
A

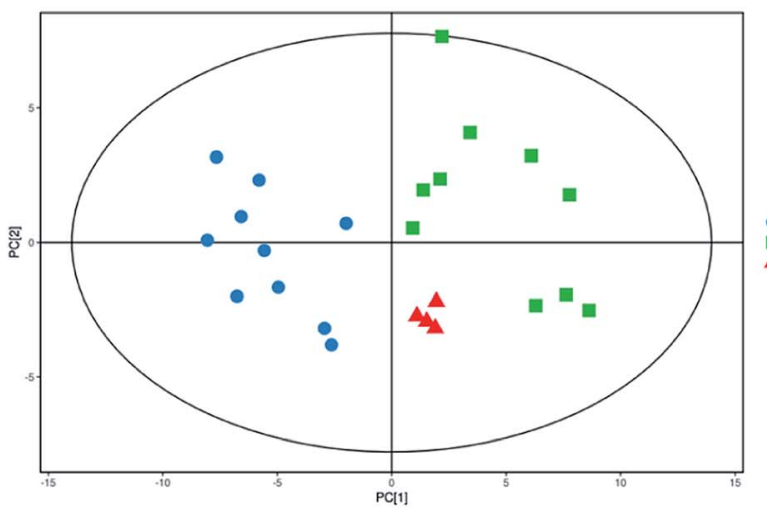

B

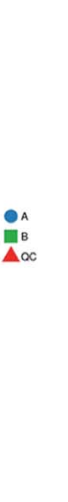

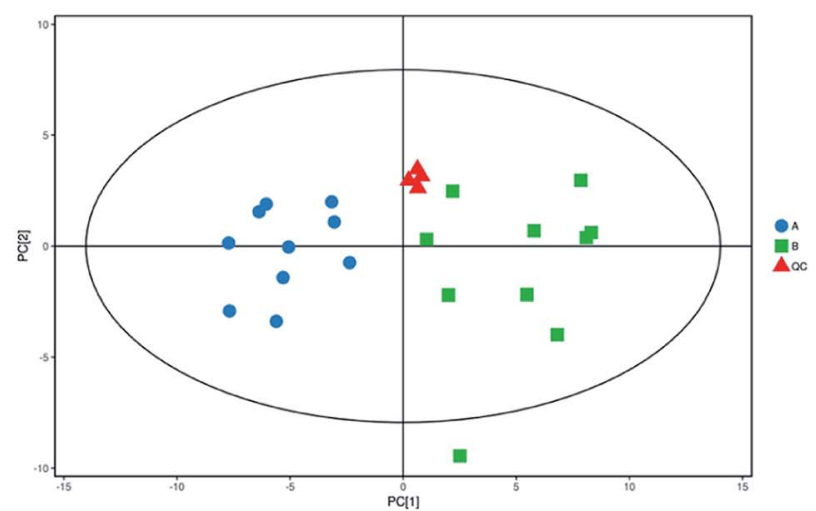

Fig. 4 Multivariate analysis of untargeted metabolomics data. PCA score plots of metabolic profile from different groups in positive ESI mode (A) and negative ESI mode (B).
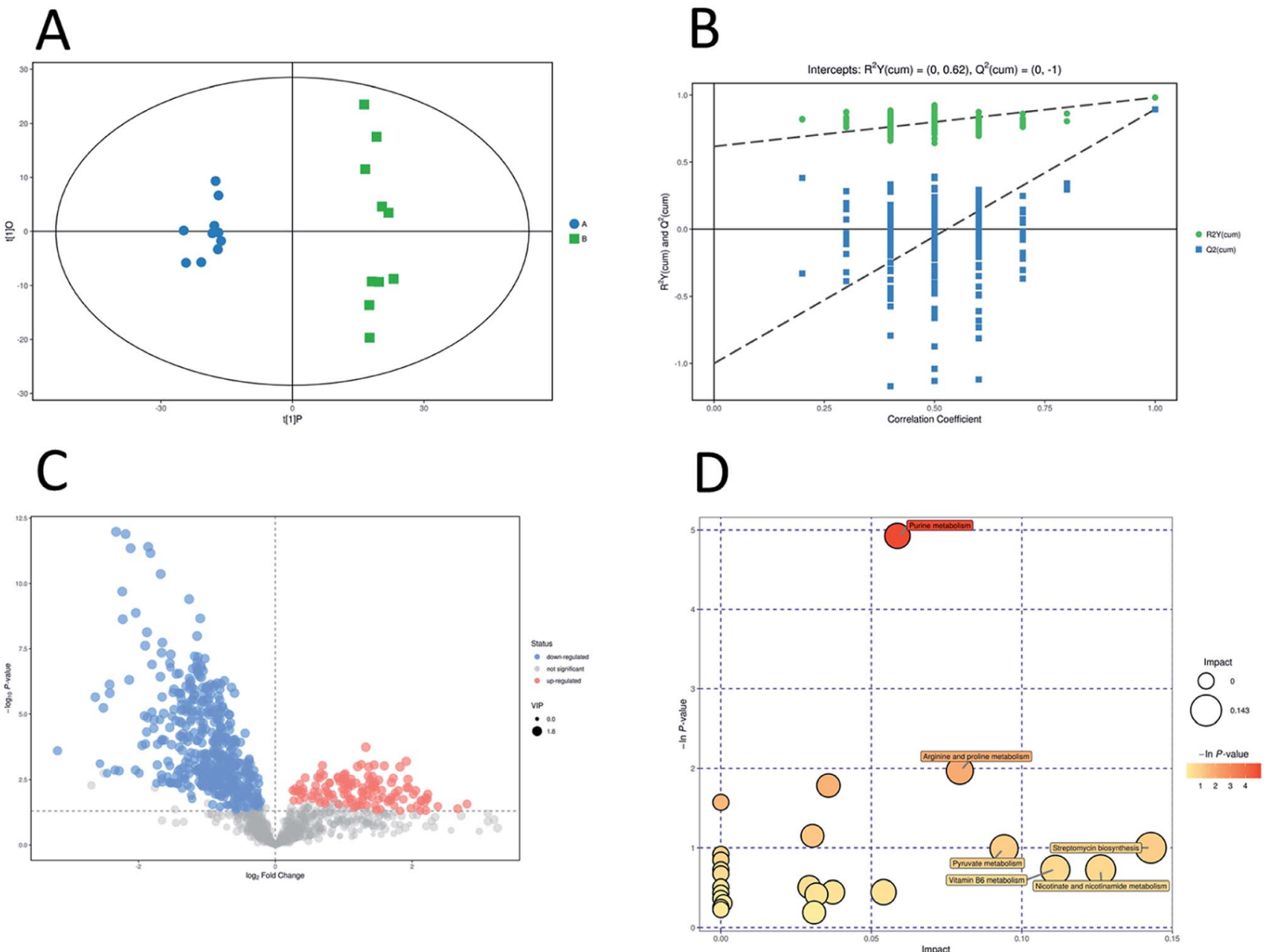

Fig. 5 Multivariate analysis of untargeted metabolomics data and pathway analysis in positive mode. OPLS-DA score plots of metabolic profiling of different groups (A). The corresponding 200 random permutations plots of the OPLS-DA models (B). Volcano plots for ESBL-EC vs. non-ESBLEC (C). The relative metabolite level is depicted according to color scale. Red represents the upregulation and blue represents the downregulation of the metabolites in ESBL-EC vs. non-ESBL-EC. Large sizes represent high VIP values of metabolites. Overview of significantly changed pathway analysis for ESBL-EC vs. non-ESBL-EC (D). The $x$-axis represents pathway enrichment. The $y$-axis represents the pathway impact. Large sizes and dark colors represent the major pathway enrichment and high pathway impact values, respectively. 

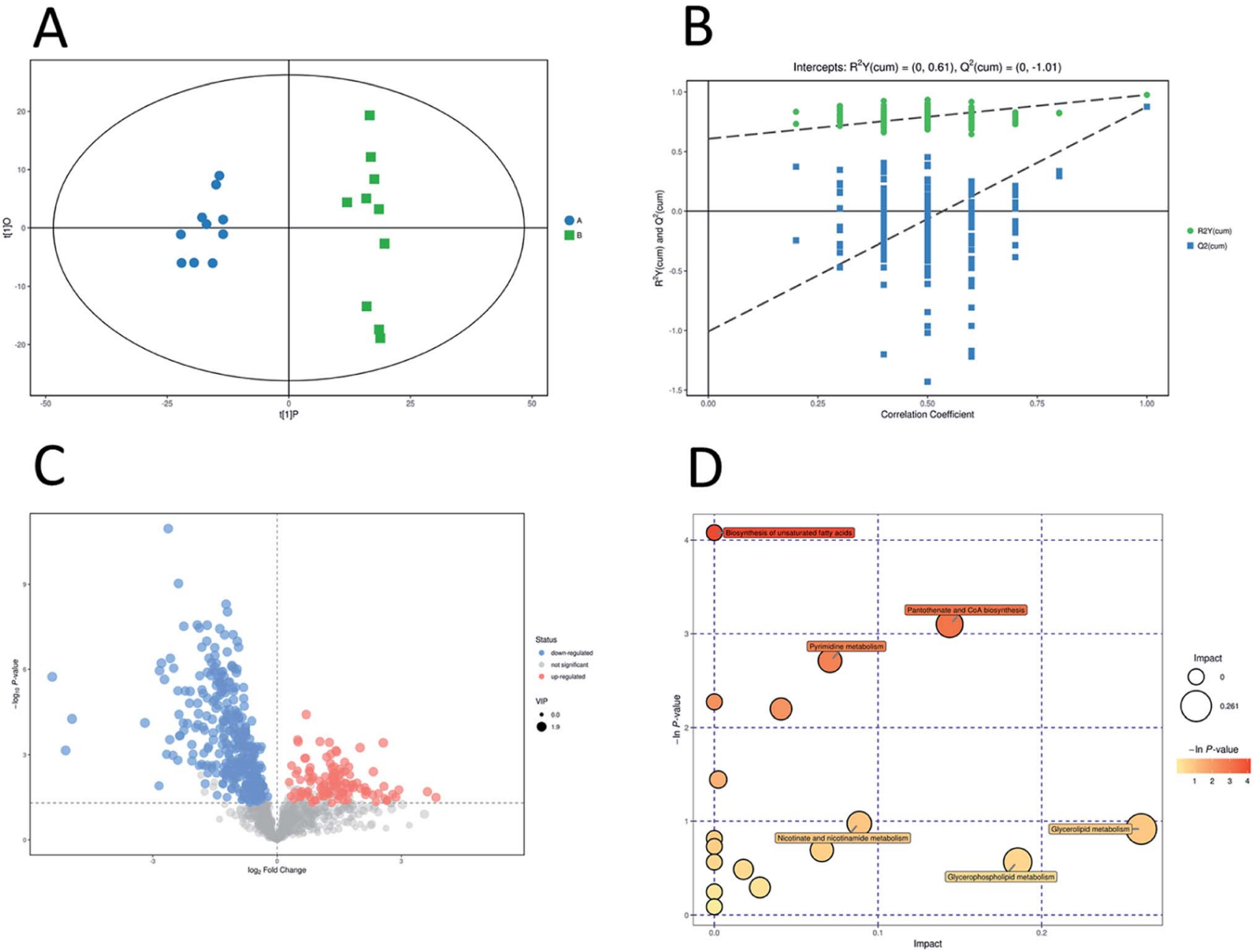

Fig. 6 Multivariate analysis of untargeted metabolomics data and pathway analysis in negative mode. OPLS-DA score plots of metabolic profiling of different groups (A). The corresponding 200 random permutations plots of the OPLS-DA models (B). Volcano plots for ESBL-EC vs. non-ESBLEC (C). The relative metabolite level is depicted according to color scale. Red represents the upregulation and blue represents the downregulation of the metabolites in ESBL-EC vs. non-ESBL-EC. Large sizes represent high VIP values of metabolites. Overview of significantly changed pathway analysis for ESBL-EC vs. non-ESBL-EC (D). The $x$-axis represents pathway enrichment. The $y$-axis represents the pathway impact. Large sizes and dark colors represent the major pathway enrichment and high pathway impact values, respectively.

Table 2 Pathway analysis of differential metabolites for ESBL-EC vs. non-ESBL-EC in positive mode

\begin{tabular}{|c|c|c|c|c|c|c|}
\hline Pathway & Total & Hits & Raw $p$ & $-\ln (p)$ & FDR & Impact \\
\hline Purine metabolism & 73 & 9 & 0.007253 & 4.9264 & 0.63099 & 0.05866 \\
\hline Arginine and proline metabolism & 41 & 4 & 0.13937 & 1.9706 & 1 & 0.0794 \\
\hline Glycerophospholipid metabolism & 23 & 2 & 0.31626 & 1.1512 & 1 & 0.03043 \\
\hline Streptomycin biosynthesis & 9 & 1 & 0.36767 & 1.0006 & 1 & 0.14286 \\
\hline Pyruvate metabolism & 26 & 2 & 0.37182 & 0.98934 & 1 & 0.09409 \\
\hline Butanoate metabolism & 18 & 1 & 0.60186 & 0.50774 & 1 & 0.02941 \\
\hline Citrate cycle (TCA cycle) & 20 & 1 & 0.64096 & 0.44479 & 1 & 0.0372 \\
\hline Propanoate metabolism & 20 & 1 & 0.64096 & 0.44479 & 1 & 0.05405 \\
\hline Glutathione metabolism & 21 & 1 & 0.65908 & 0.41692 & 1 & 0.03175 \\
\hline $\begin{array}{l}\text { Valine, leucine and isoleucine } \\
\text { biosynthesis }\end{array}$ & 26 & 1 & 0.73704 & 0.30511 & 1 & 0.00085 \\
\hline
\end{tabular}


Table 3 Pathway analysis of differential metabolites for ESBL-EC vs. non-ESBL-EC in negative mode

\begin{tabular}{|c|c|c|c|c|c|c|}
\hline Pathway & Total & Hits & Raw $p$ & $-\ln (p)$ & FDR & Impact \\
\hline Pantothenate and CoA biosynthesis & 23 & 3 & 0.045 & 3.1011 & 1 & 0.14377 \\
\hline Pyrimidine metabolism & 44 & 4 & 0.066497 & 2.7106 & 1 & 0.07066 \\
\hline Pyruvate metabolism & 26 & 2 & 0.23588 & 1.4444 & 1 & 0.00239 \\
\hline Nicotinate and nicotinamide metabolism & 13 & 1 & 0.37763 & 0.97384 & 1 & 0.08859 \\
\hline Glycerolipid metabolism & 14 & 1 & 0.40008 & 0.91609 & 1 & 0.26087 \\
\hline $\begin{array}{l}\text { Valine, leucine and isoleucine } \\
\text { biosynthesis }\end{array}$ & 26 & 1 & 0.6151 & 0.48597 & 1 & 0.0178 \\
\hline Galactose metabolism & 37 & 1 & 0.745 & 0.29438 & 1 & 0.02786 \\
\hline
\end{tabular}

mobile phase consisted of $25 \mathrm{mM} \mathrm{NH}_{4} \mathrm{OAc}$ and $25 \mathrm{mM} \mathrm{NH}_{4} \mathrm{OH}$ in water $(\mathrm{pH}=9.75)(\mathrm{A})$ and acetonitrile $(\mathrm{B})$ was carried with elution gradient as follows: $0 \mathrm{~min}, 95 \% \mathrm{~B} ; 7 \mathrm{~min}, 65 \% \mathrm{~B} ; 9 \mathrm{~min}$, $40 \% \mathrm{~B} ; 9.1 \mathrm{~min}, 95 \% \mathrm{~B} ; 12 \mathrm{~min}, 95 \% \mathrm{~B}$, which was delivered at $0.5 \mathrm{~mL} \mathrm{~min}^{-1}$. The injection volume was $2 \mu \mathrm{L}$. The Triple TOF mass spectrometer was used for its ability to acquire MS/MS spectra on an information-dependent basis (IDA) during an LC/MS experiment. In this mode, the acquisition software (Analyst TF 1.7, AB Sciex) continuously evaluates the full scan survey MS data as it collects and triggers the acquisition of MS/ MS spectra depending on preselected criteria. In each cycle, 12 precursor ions whose intensity greater than 100 were chosen for fragmentation at collision energy (CE) of $30 \mathrm{~V}$ (15 MS/MS events with product ion accumulation time of $50 \mathrm{~m} \mathrm{~s}$ each). ESI source conditions were set as following: ion source gas 1 as 60 psi, ion source gas 2 as $60 \mathrm{psi}$, curtain gas as $35 \mathrm{psi}$, source temperature $650{ }^{\circ} \mathrm{C}$, Ion Spray Voltage Floating (ISVF) $5000 \mathrm{~V}$ or $-4000 \mathrm{~V}$ in positive or negative modes, respectively. Untargeted metabolomics were then subjected to the data processing and metabolite annotation as described in the ESI. $\dagger^{20,21}$

2.4.3. UPLC-MRM-MS (ultra-performance liquid chromatography multiple reaction monitoring mass spectrometry) analysis. For the targeted metabolomics analysis, the UHPLC separation was carried out using an Agilent 1290 Infinity II series UHPLC System (Agilent Technologies), equipped with a Waters ACQUITY UPLC BEH Amide column $(100 \times 2.1 \mathrm{~mm}$, $1.7 \mu \mathrm{m})$. The mobile phase A was $1 \%$ formic acid in water, and the mobile phase $\mathrm{B}$ was acetonitrile. The elution gradient was shown in Table S6. $\dagger$ The column temperature was set at $35^{\circ} \mathrm{C}$. The auto-sampler temperature was set at $4{ }^{\circ} \mathrm{C}$ and the injection
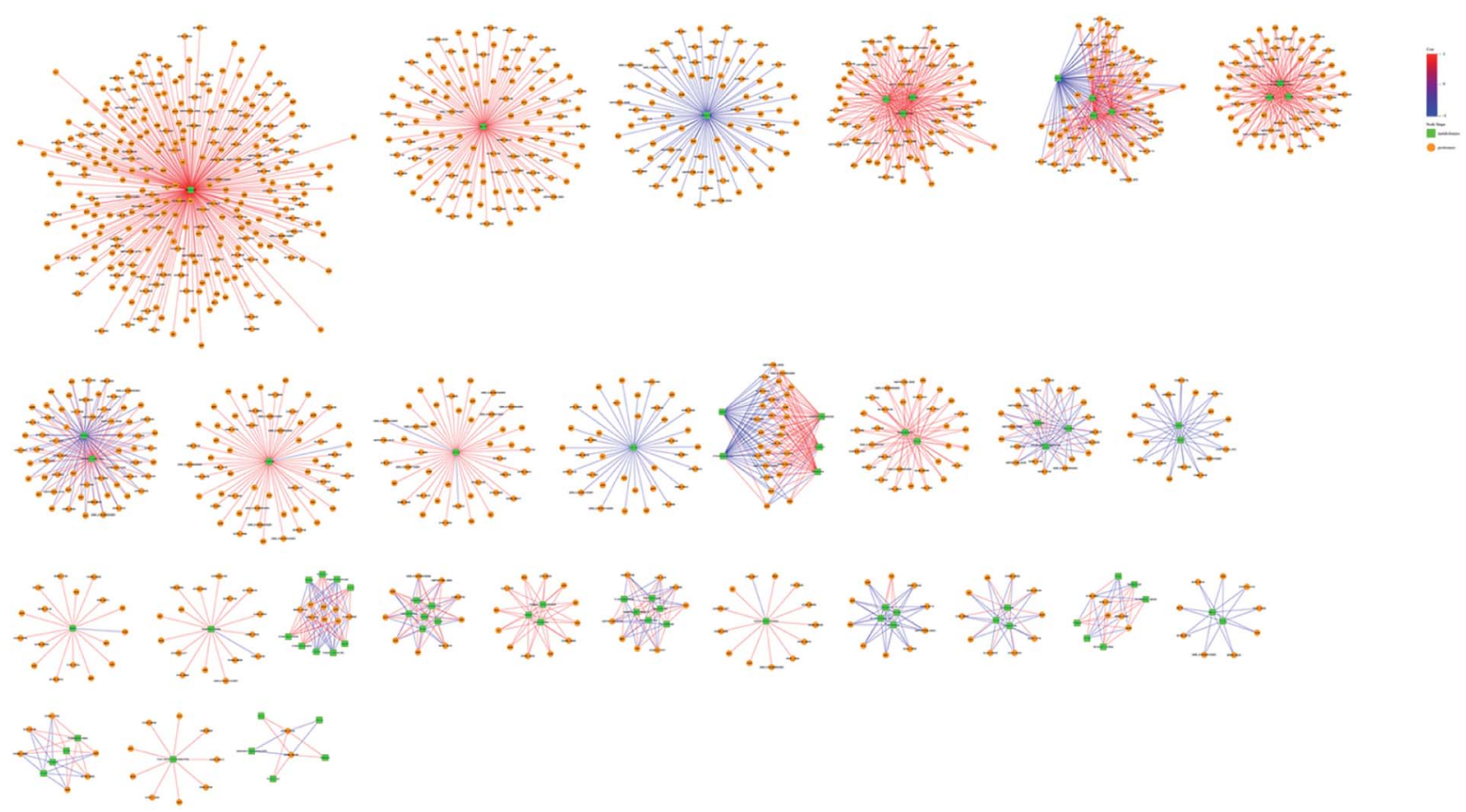

Fig. 7 Metabolite-protein interaction network based on the differential metabolites and DEPs for ESBL-EC vs. non-ESBL-EC in positive mode. The green squares represent differential metabolites. The yellow circles represent differential protein genes. The red line represents a positive correlation between the differential metabolites and the differential protein genes. The blue line represents a negative correlation. 

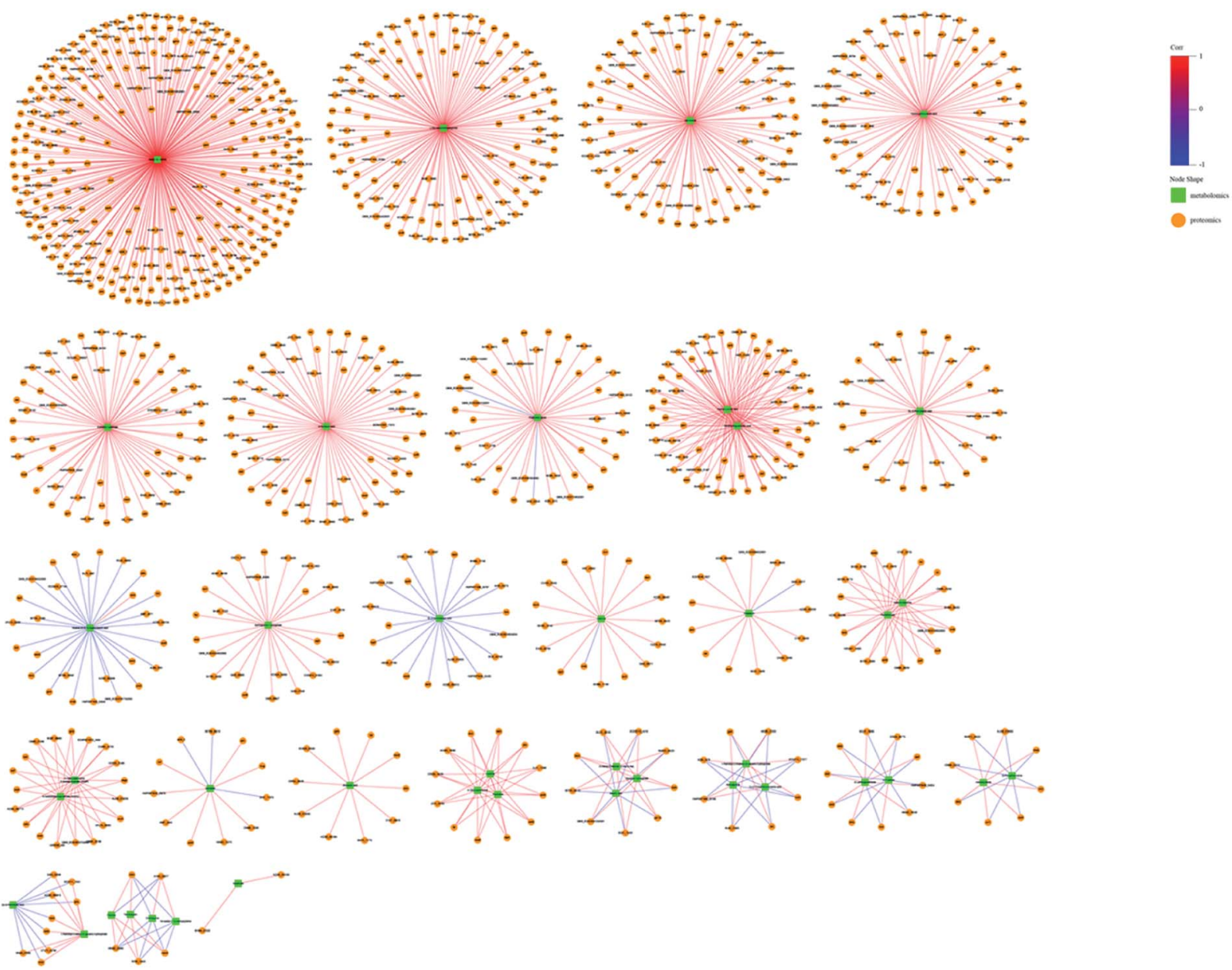

Fig. 8 Metabolite-protein interaction network based on the differential metabolites and DEPs for ESBL-EC vs. non-ESBL-EC in negative mode. The green squares represent differential metabolites. The yellow circles represent differential protein genes. The red line represents a positive correlation between the differential metabolites and the differential protein genes. The blue line represents a negative correlation.

volume was $1 \mu \mathrm{L}$. An Agilent 6460 triple quadrupole mass spectrometer (Agilent Technologies), equipped with an AJS electrospray ionization (AJS-ESI) interface, was applied for assay development. Typical ion source parameters were: capillary voltage $=+4000 /-3500 \mathrm{~V}$, nozzle voltage $=+500 /-500 \mathrm{~V}$, gas $\left(\mathrm{N}_{2}\right)$ temperature $=300{ }^{\circ} \mathrm{C}$, gas $\left(\mathrm{N}_{2}\right)$ flow $=5 \mathrm{~L} \mathrm{~min}^{-1}$, sheath gas $\left(\mathrm{N}_{2}\right)$ temperature $=250{ }^{\circ} \mathrm{C}$, sheath gas flow $=11 \mathrm{~L} \mathrm{~min}^{-1}$, nebulizer $=45 \mathrm{psi}$. The MRM parameters for each of the targeted analytes were optimized using flow injection analysis, by injecting the standard solutions of the individual analytes, into the API source of the mass spectrometer. Several most sensitive transitions were used in the MRM scan mode to optimize the collision energy for each Q1/Q3 pair (Table S7†). Among the optimized MRM transitions per analyte, the Q1/Q3 pairs that showed the highest sensitivity and selectivity were selected as 'quantifier' for quantitative monitoring. The additional transitions acted as 'qualifier' for the purpose of verifying the identity of the target analytes. Agilent MassHunter Work Station Software (B.08.00, Agilent Technologies) was employed for MRM data acquisition and processing. Calibration curves LOD (limit of detection) and LOQ (limit of quantitation), precision and accuracy were established to guarantee the analysis stability, details see in the ESI. $\dagger$

\subsection{Statistical analysis}

The data were analyzed with student's $T$ test by using Excel 2013. A $p$-value of $<0.05$ was considered significant.

\section{Results}

\subsection{TMT quantitative proteomics analysis}

The 1553 DEPs (differentially expressed proteins) (fold change < 0.83 or fold change $>1.2 ; p$-value $<0.05$ ) were identified by TMT MS; among the DEPs, 18 were upregulated and 1535 were downregulated in the ESBL-EC (Fig. 1A, B and S1 $\dagger$ ). The detailed results of the DEPs are shown in Table S1. $\dagger$

\subsection{Function analysis results}

Systematic and integrative analysis was performed using DAVID to gain a comprehensive understanding of the DEPs. ${ }^{22}$ The properties of the genes and gene products of the DEPs were classified into three categories: GO_BP (GO Term Biological 

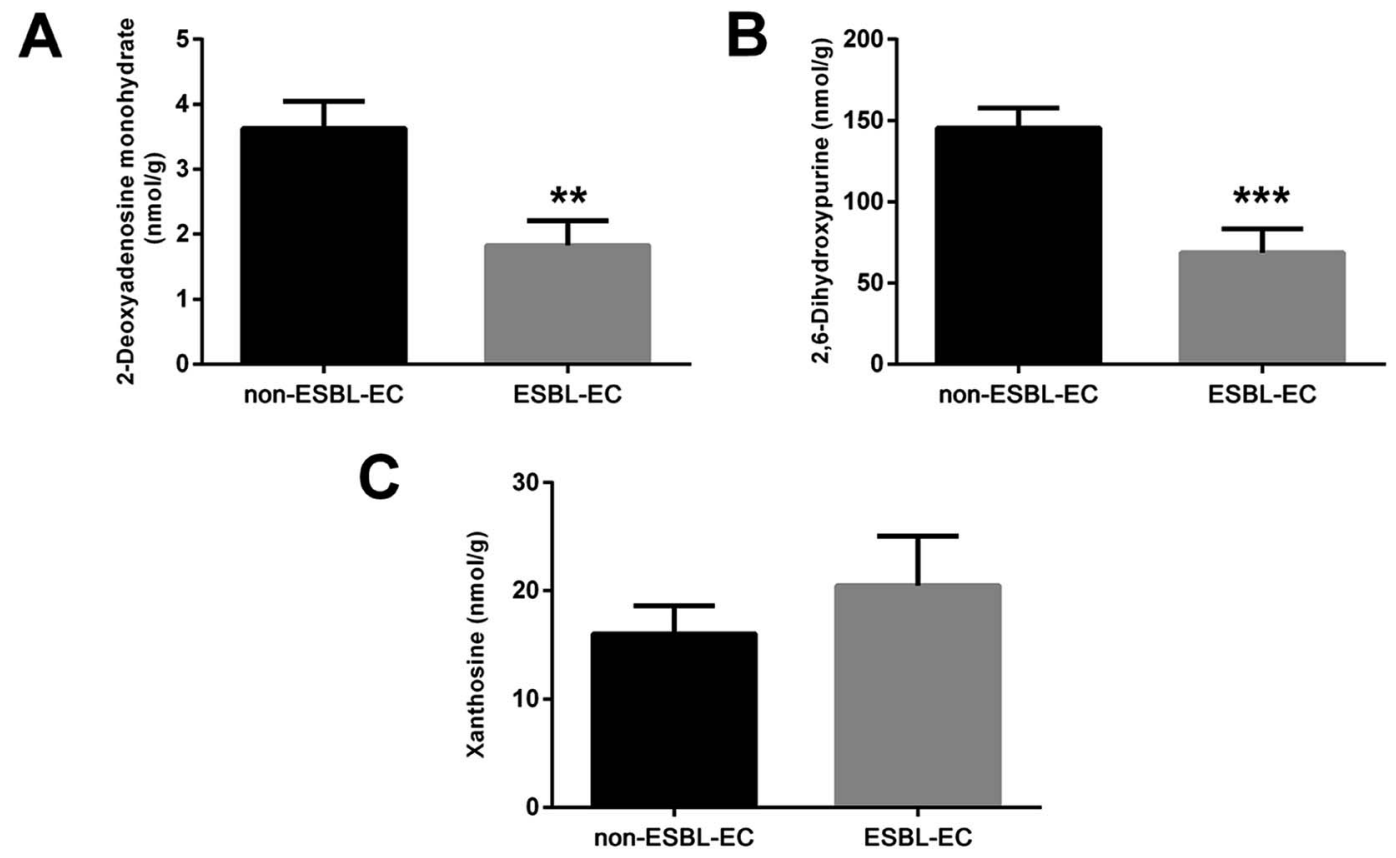

Fig. 9 Target metabolomics analysis by using MRM for three differential metabolites for ESBL-EC vs. non-ESBL-EC: (A) 2-deoxyadenosine monohydrate, (B) 2,6-dihydroxypurine, and (C) xanthosine. The values are presented as mean $\pm \mathrm{SD}, n=10 . * * p$-value $<0.01$, and $* * * p$-value $<$ 0.001 and compared with the non-ESBL-EC group.

Process), GO_CC (Cellular Component), and GO_MF (Molecular Function). The classifications were used to identify the potential affected pathways. The DEPs were significantly enriched in 53
GO terms ( $p$-value $<0.05$ ). Among them, 29 were BP terms, 7 were CC terms, and 17 were MF terms (Table S2 $\dagger$ ). The 30 most representative and markedly enriched GO terms within the

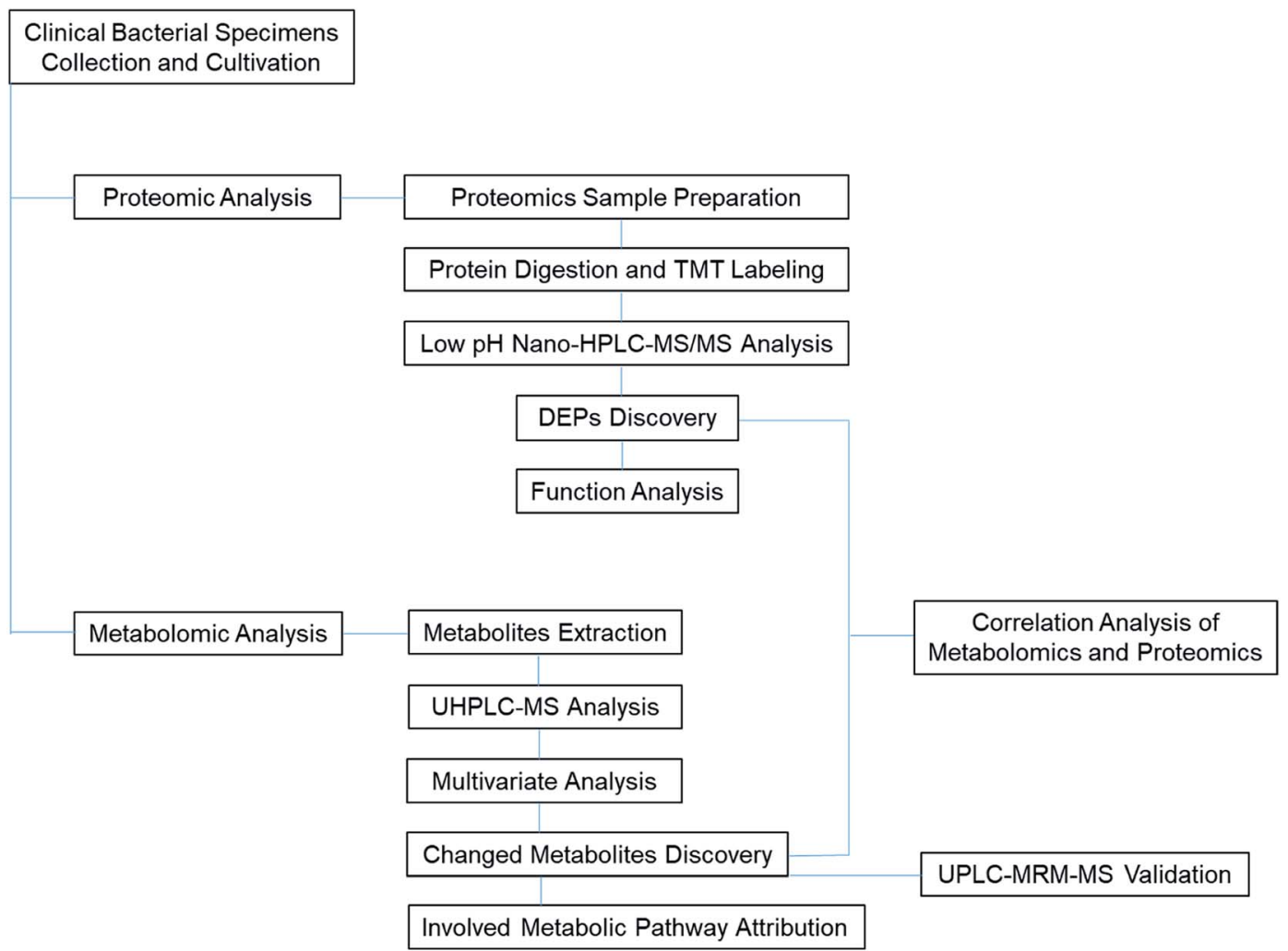

Fig. 10 TOC graph for illustration of the proteomic and metabolomic analytical process of clinical ESBL specimens. 
three main functional modules are shown in Fig. 1C. The leading seven BP terms were "translation", "transcription antitermination", "glycolytic process", "isopentenyl diphosphate biosynthetic process, methylerythritol 4-phosphate pathway," "negative regulation of translational initiation", "nucleobasecontaining small molecule interconversion" and "Ubiquinone biosynthetic process" (Fig. 2).

In addition, significantly enriched metabolic pathways of DEPs were identified using the KEGG database to determine the systemic difference between ESBL-EC and non-ESBL-EC. ${ }^{23} \mathrm{~A}$ total of 82 significant systemically changed KEGG pathways were detected (Table S3†). PPI network analyses are important in the discovery of the variation tendency of DEPs in proteomics analysis, and they are helpful in targeting the key node between DEPs.

We constructed the PPI of $E$. coli by using the STRING database ${ }^{23}$ to uncover the potential protein interaction of DEPs and the biological functions in the bacterial cellular processes for ESBL-EC (Fig. 3). Moreover, the PPI networks analysis was used to obtain different central nodes, including hisA, glcE, soda, and GroL.

\subsection{Multivariate analysis of UPLC-QTof/MS data for the metabolomics study}

Unsupervised pattern recognition PCA (principal component analysis) was conducted to visualize clearly the separation of the metabolites between ESBL-EC and non-SBL-EC (Fig. 4). QC injections were closely clustered in the PCA score plots in both positive mode $\left(R^{2} X=0.525\right)$ and negative mode $\left(R^{2} X=0.531\right)$. This study has verified the stability of the UPLC-QTof/MS system.

In addition, OPLS-DA (orthogonal projections to latent structures discriminant analysis) was performed to remove the descriptor variables that were not correlated to the property variables and determine the differential metabolite profiles that was responsible for the classification between the two groups (Fig. 5A and 6A). The $R^{2} X$ and $R^{2} Y$ of the OPLS-DA model were 0.364 and 0.982 for the ESI+ mode and 0.352 and 0.976 for the ESI- mode, respectively. Subsequently, seven-fold cross validation was implemented and applied with 200 random permutations to validate the OPLS-DA models. All blue $Q^{2}$ values at the left part were lower than the original points on the right part (Fig. 5B and 6B). This finding indicates that the original models are robust and have no overfitting.

\subsection{Potential marker identification and the involved metabolic pathway attribution}

The volcano plots, which were produced on the basis of OPLSDA, depict the contribution of the variables between the two groups (Fig. 5C and 6C). MetaboAnalyst was used to search for the pathways of the changed metabolites. The pathway analysis based on the potential biomarkers showed 18 different metabolic pathways between the two groups (Fig. 5D, 6D; Tables 2 and 3).

\subsection{Correlation analysis of metabolomics and proteomics}

The omic-domain data integration for ESBL-EC was obtained by subjecting the content information of the DEPs and differential metabolites to relevant calculations by using the Spearman algorithm. The $p$-value of the correlation matrix was used for the follow-up analysis. A correlation network graph (Fig. 7 and 8) was constructed on the basis of the data that could meet both the correlation coefficient of $>0.9$ and the $p$-value of $<0.05$. In the figure, the green squares represent differential metabolites, the yellow circles represent the differential protein genes, the red line represents a positive correlation between the differential metabolites and the differential protein genes, and the blue line represents a negative correlation. Tables S8-S11† show all of the binary relations of the subnetworks.

\subsection{Target metabolomics analysis}

The drug resistance mechanism of ESBL-EC was further investigated, then the non-target metabolomics results were verified in this study. The sequential research was focused on the differential metabolites in the purine metabolism pathway (eco00230-E. coli K-12 MG1655). Three differential metabolites (2-deoxyadenosine monohydrate, 2,6-dihydroxypurine, and xanthosine) in the purine metabolism pathway were selected and subjected to target metabolomics analysis assay development by using UHPLC-QQQ-MS (MRM). As shown in Fig. S2, $\dagger$ the baseline separation of the metabolites can be successfully obtained, and the retention time and peak shapes of all of the analytes present a good match between the standard solution and the real sample, i.e., 2-deoxyadenosine monohydrate (RT, $2.36 \mathrm{~min}$ ), 2,6-dihydroxypurine (RT, $2.28 \mathrm{~min}$ ), and xanthosine (RT, $3.30 \mathrm{~min}$ ). Appropriate linear range with high linearity and low LOD and LOQ facilitate sensitive and selective analysis for the metabolites from biological samples. The LODs of the three metabolites were 1.22-19.53 nM, while the LOQs were 2.44$39.06 \mathrm{nM}$, with high linearity $\left(R^{2}>0.9997\right)$. The details of the calibration curves are shown in Table S4. $\dagger$ The five repeated injections presented a recovery from $93.5 \%$ to $104.2 \%$, and the RSD of the targeted matabolites was less than $3.1 \%$ (Table S5 $\dagger$ ), which accords with the quantification of the targeted metabolomics analysis.

Then the rapid MRM based target metabolomics analysis was successfully applied to absolutely quantify the changed metabolites in the clinical bacterial samples. The MRM result (Fig. 9) showed that the contents of the 2-deoxyadenosine monohydrate and 2,6-dihydroxypurine were downregulated ( $p$ value $<0.01)$. This finding is consistent with the non-target metabolomics analysis. However, the xanthosine was relatively overexpressed in ESBL-EC ( $p$-value $>0.05$ ), a finding inconsistent with the non-target metabolomics analysis.

\section{Discussion}

Antibiotics are commonly used as the first line of action in the treatment or prevention of certain bacterial infections. ${ }^{24}$ However, antibiotics do not work for patients infected with the ESBL-producing $E$. coli and thus a worldwide important public 
health concern. ${ }^{25}$ As discussed earlier, the antibiotic resistance and ESBL production capacity of $E$. coli remains poorly understood. ${ }^{18}$ Here, we examined for the first time the proteomics and metabolomics differences between ESBL-EC and non-ESBL-EC to clarify the potential mechanism of the ESBL-EC's antibiotic resistance and provide a scientific evidence for the discovery of novel targets for groundbreaking therapies.

Among the 18 different metabolic pathways classified between ESBL-EC and non-ESBL-EC, the purine metabolism pathway showed a high enriched degree, as characterized by the lowest $\ln p$-value and dark bubble in the bubble plots. Purine metabolism was found to be correlated to the drug resistance of parasites and the drug resistance and tumor relapse of childhood ALL in previous work. ${ }^{26,27}$ More recently, a comparative metabolomics study based on GC-MS showed that the metabolic profiles of purine metabolism fluctuates in multi-drugresistant $E$. coli strains; this result from previous work ${ }^{\mathbf{1 8}}$ agrees with our current findings. In addition, in using metabolomics and proteomics correlation analysis, we found drug resistance affects the metabolite and protein profiles of $E$. coli, as many central metabolite nodes were obtained, including 2-deoxyadenosine monohydrate, 2,6-dihydroxypurine, and xanthosine, which have significant correlations with DEPs.

Considering the potential significance of purine metabolism for the drug resistance of ESBL-EC, target metabolomics analysis was performed using MRM. We selected three different metabolites (2-deoxyadenosine monohydrate, 2,6-dihydroxypurine, and xanthosine) in the purine metabolism pathway for further quantitative verification analysis, with comprehensive consideration of the MS2 score, $p$-value, VIP, and metaboliteprotein interactions in the correlation analysis. The MRM analytical results coincided with the non-targeted metabolomics data, suggesting the reliability of our analytical method. Moreover, the above metabolites may be involved in the drug resistance of the bacteria in ESBL-EC.

DEPs may play important roles in the antibiotic resistance and ESBL production capacity of ESBL-EC. The present work has provided scientific data for the metabolic and protein level characteristics and involved important pathways of clinical $\beta$ lactamase enzyme-producing bacteria, which revealed the potential mechanism for the antibiotic resistance of ESBL-EC.

The main purpose of the experiment is using the proteomic and metabolomics to explore the underline mechanism of extended-spectrum $\beta$-lactamase production in $E$. coli. On the basic of above study, our future work is going on, such as performing site-by-point editing of the critical node protein-related genes or the purine metabolism pathway in ESBL-EC by using gene editing tools, such as the CRISPR/Cas9 system, based on the above research results. We also plan to analyze the bacterial virulence, extended-spectrum $\beta$-lactamase activity, and antibiotic resistance of genetically engineered $E$. coli. Another plan is to construct a gRNA recombination vector-based vaccine carrier bacteria with the aim of eliminating or weakening the activity of $\beta$-lactam drug resistance genes. We also intend to test the efficacy of the vaccine carrier bacteria in vivo. Briefly, mice will be pre-stimulated with the vaccine carrier bacteria, then we shall establish the mouse infection model by using clinical ESBL-EC, and finally, we will observe the therapeutic effect of $\beta$-lactam drugs on the infection of mice.

\section{Conclusion}

This basic research has shown how metabolic and proteomics alterations can manifest in ESBL-EC. The untargeted metabolomics result was also confirmed by the LC-MRM-MS analysis. With the aid of metabolomics and proteomics correlation bioinformatics analysis, we obtained many central metabolite nodes and their corresponding DEPs. The findings can help in our future study of the antibiotic resistance mechanism in ESBL-EC and the corresponding research and development of biomedicine-based target therapy (Fig. 10).

\section{Author contributions}

Data curation: Bingjie Lai, Chang Tian; investigation: Jiaying Liu; project adminstration: He Ma, Yufen Jin; resources: Ke Wang; software: Bingjie Lai; supervision: Ke Wang; writingoriginal draft: He Ma; writing-review: Ke Wang.

\section{Conflicts of interest}

The authors declare no conflict of interest.

\section{Acknowledgements}

This work has been supported by Social Development Project of Jilin Science and Technology Department (20190303162SF), Advanced Program of Jilin Science and Technology Department (20191102012YY), and Natural Science Foundation of Jilin Province (20180101103JC).

\section{References}

1 O. Tenaillon, D. Skurnik, B. Picard and E. Denamur, The population genetics of commensal Escherichia coli, Nat. Rev. Microbiol., 2010, 8(3), 207-217.

2 C. I. Kang, M. K. Cha, S. H. Kim, K. S. Ko, Y. M. Wi, D. R. Chung, K. R. Peck, N. Y. Lee and J. H. Song, Clinical and molecular epidemiology of community-onset bacteremia caused by extended-spectrum beta-lactamaseproducing Escherichia coli over a 6-year period, J. Kor. Med. Sci., 2013, 28(7), 998-1004.

3 U. Klümper, M. Recker, L. Zhang, X. Yin, T. Zhang, A. Buckling and W. H. Gaze, Selection for antimicrobial resistance is reduced when embedded in a natural microbial community, ISME J., 2019, 2927-2937.

4 X. Liu, J. Wang, M. Chen, R. Che, W. Ding, F. Yu, Y. Zhou, W. Cui, X. Xiaoxu, B. O. God'spower and Y. Li, Comparative proteomic analysis reveals drug resistance of Staphylococcus xylosus ATCC700404 under tylosin stress, BMC Vet. Res., 2019, 15(1), 224.

5 J. Rodriguez-Bano, M. D. Navarro, L. Romero, M. A. Muniain, M. de Cueto, M. J. Rios, J. R. Hernandez and A. Pascual, Bacteremia due to extended-spectrum beta -lactamase- 
producing Escherichia coli in the CTX-M era: a new clinical challenge, Clin. Infect. Dis., 2006, 43(11), 1407-1414.

6 M. Zampieri, T. Enke, V. Chubukov, V. Ricci, L. Piddock and U. Sauer, Metabolic constraints on the evolution of antibiotic resistance, Mol. Syst. Biol., 2017, 13(3), 917.

7 D. K. Derewacz, C. R. Goodwin, C. R. McNees, J. A. McLean and B. O. Bachmann, Antimicrobial drug resistance affects broad changes in metabolomic phenotype in addition to secondary metabolism, Proc. Natl. Acad. Sci. U.S.A., 2013, 110(6), 2336-2341.

8 G. Monni, F. Murgia, V. Corda, C. Peddes, A. Iuculano, L. Tronci, A. Balsamo and L. Atzori, Metabolomic Investigation of beta-Thalassemia in Chorionic Villi Samples, J. Clin. Med., 2019, 8(6), 798.

9 H. T. Lin, M. L. Cheng, C. J. Lo, G. Lin, S. F. Lin, J. T. Yeh, H. Y. Ho, J. R. Lin and F. C. Liu, ${ }^{1} \mathrm{H}$ Nuclear Magnetic Resonance (NMR)-Based Cerebrospinal Fluid and Plasma Metabolomic Analysis in Type 2 Diabetic Patients and Risk Prediction for Diabetic Microangiopathy, J. Clin. Med., 2019, 8(6), 874.

10 C. J. Lo, H. Y. Tang, C. Y. Huang, C. M. Lin, H. Y. Ho, M. S. Shiao and M. L. Cheng, Metabolic Signature Differentiated Diabetes Mellitus from Lipid Disorder in Elderly Taiwanese, J. Clin. Med., 2018, 8(1), 13.

11 K. Wanichthanarak, J. F. Fahrmann and D. Grapov, Genomic, Proteomic, and Metabolomic Data Integration Strategies, Biomark. Insights, 2015, 10(Suppl 4), 1-6.

12 D. Du, X. Wang-Kan, A. Neuberger, H. W. van Veen, K. M. Pos, L. J. V. Piddock and B. F. Luisi, Multidrug efflux pumps: structure, function and regulation, Nat. Rev. Microbiol., 2018, 16(9), 523-539.

13 W. Wei, H. Yan, J. Zhao, H. Li, L. Zhou, H. Guo, X. Wang, Y. Zhou, X. Zhang, J. Zeng, T. Chen and L. Zhou, Multiomics comparisons of $\mathrm{p}$-aminosalicylic acid (PAS) resistance in folC mutated and un-mutated Mycobacterium tuberculosis strains, Emerging Microbes Infect., 2019, 8(1), 248-261.

14 J. Zhao, W. Wei, H. Yan, Y. Zhou, Z. Li, Y. Chen, C. Zhang, J. Zeng, T. Chen and L. Zhou, Assessing Capreomycin Resistance on tlyA Deficient and Point Mutation (G695A) Mycobacterium Tuberculosis Strains Using Multi-Omics Analysis, Int. J. Med. Microbiol. Suppl., 2019, 309(7), 151323.

15 A. Marrugal, I. Ferrer, M. D. Pastor, L. Ojeda, A. QuintanalVillalonga, A. Carnero, S. Molina-Pinelo and L. Paz-Ares, Impact of Heat Shock Protein 90 Inhibition on the Proteomic Profile of Lung Adenocarcinoma as Measured by Two-Dimensional Electrophoresis Coupled with Mass Spectrometry, Cells, 2019, 8(8), 806.

16 L. Yuan, J. Wang, S. Xie, M. Zhao, L. Nie, Y. Zheng, S. Zhu, J. Hou, G. Chen and C. Wang, Comparative Proteomics
Indicates That Redox Homeostasis Is Involved in Highand Low-Temperature Stress Tolerance in a Novel Wucai (Brassica campestris L.) Genotype, Int. J. Mol. Sci., 2019, 20(15), 3760.

17 M. Mann and N. L. Kelleher, Precision proteomics: The case for high resolution and high mass accuracy, Proc. Natl. Acad. Sci. U.S.A., 2008, 105(47), 18132-18138.

18 Y. Lin, W. Li, L. Sun, Z. Lin, Y. Jiang, Y. Ling and X. Lin, Comparative metabolomics shows the metabolic profiles fluctuate in multi-drug resistant Escherichia coli strains, $J$. Proteomics, 2019, 207, 103468.

19 L. Li, Q. Wu, Y. Wang, S. Morteza, Z. Ban, X. Zhang, H. Lu, D. Li, J. Yan, J. Limwachiranon and Z. Luo, Systematically quantitative proteomics and metabolite profiles offer insight into fruit ripening behavior in fragaria $\times$ ananassa, RSC Adv., 2019, 9, 14093-14108.

$20 \mathrm{H}$. Yao, P. Yu and C. Jiang, Metabolomics-driven identification of perturbations in amino acid and sphingolipid metabolism as therapeutic targets in a rat model of anorexia nervosa disease using chemometric analysis and a multivariate analysis platform, RSC Adv., 2020, 10, 4928.

21 N. Yang, H. Wang, H. Lin, J. Liu, B. Zhou, X. Chen, C. Wang, J. Liu and P. Li, Comprehensive metabolomics analysis based on uplc-q/tof-mse and the anti-copd effect of different parts of celastrus orbiculatus thunb, RSC Adv., 2020, 10, 8396.

22 B. Anindya, M. Biswajit and S. J. Susanna, Global transcriptome analysis reveals distinct bacterial response towards soluble and surface-immobilized antimicrobial peptide (lasioglossin- III), RSC Adv., 2015, 5(96), 7871278718.

23 A. Bilal, B. Madiha, N. M. Atif, K. Mohsin and R. M. Hidayat, Proteomics: Technologies and Their Applications, $J$. Chromatogr. Sci., 2016, 1-15.

24 R. J. Fair and Y. Tor, Antibiotics and bacterial resistance in the 21st century, Perspect. Med. Chem., 2014, 6, 25-64.

25 M. Melzer and I. Petersen, Mortality following bacteraemic infection caused by extended spectrum beta-lactamase (ESBL) producing E. coli compared to non-ESBL producing E. coli, J. Infect., 2007, 55(3), 254-259.

26 W. B. Parker and M. C. Long, Purine metabolism in Mycobacterium tuberculosis as a target for drug development, Curr. Pharmaceut. Des., 2007, 13(6), 599-608.

27 H. Li, B. Li, F. Yang, C. Duan, Y. Bai, J. J. Yang, J. Chen, A. von Stackelberg, H. Chen, J. Tang, A. A. Ferrando, J. Zhang, S. Wang, R. Kirschner-Schwabe and B.-B. S. Zhou, De Novo Purine Biosynthesis in Drug Resistance and Tumor Relapse of Childhood ALL, Blood, 2015, 126(23), 2627. 